\title{
Coagulation markers in patients with coronary artery disease
}

\author{
Tetiana Marynenko, ${ }^{1}$ Tetiana Halenova, ${ }^{1}$ Nataliia Raksha, ${ }^{1}$ Tetiana Vovk, ${ }^{1}$ Yuliya Tyravska, ${ }^{2}$ \\ Olexii Savchuk, ${ }^{1}$ Liudmyla Ostapchenko ${ }^{1}$ \\ ${ }^{1}$ Taras Shevchenko National University of Kyiv, Kyiv; ${ }^{2}$ Bogomolets National Medical University, Kyiv, Ukraine
}

\begin{abstract}
Coronary Artery Disease (CAD) is a major public health problem all over the world, and although diagnostic and treatment approaches are improving, the majority of individuals who suffer CAD will not survive. Therefore, it is a clinical demand to find out new biomarkers for identification of CAD in the earlier stage. The purpose of this study was to determine whether qualitative and quantitative alterations of plasma Soluble Fibrin Monomer Complexes (SFMCs) are associated with cardiovascular events. Additionally, some other well-known biomarkers of hypercoagulation state, such as fibrinogen, and prothrombin were analyzed in plasma of patients with different coronary artery pathologies. We found that plasma SFMC concentrations were remarkably increased in all tested CAD groups. Moreover, the development of CAD was accompanied by the changes in
\end{abstract}

Correspondence: Tetiana Halenova, Educational and Scientific Center "Institute of Biology and Medicine", Taras Shevchenko National University of Kyiv, 64/13, Volodymyrska Street, 01601 Kyiv, Ukraine. E-mail: galenovatanya@gmail.com

Key words: Coronary artery disease; soluble fibrin monomer complexes; fibrinogen; prothrombin; vitamin K-dependent proteins, size exclusion chromatography

Conflict of interest: The authors declare no conflict of interest.

Ethics approval and consent to participate: The Ethics Committee of both the Kyiv City Hospital \#12 and Taras Sevchenko University of Kyiv approved this study. The study is conformed with the Helsinki Declaration of 1964, as revised in 2013, concerning human and animal rights. All patients participating in this study signed a written informed consent form for participating in this study.

Informed consent: Written informed consent was obtained from a legally authorized representative(s) for anonymized patient information to be published in this article.

Received for publication: 28 October 2021.

Revision received: 15 February 2022.

Accepted for publication: 22 February 2022.

${ }^{\circ}$ Copyright: the Author(s), 2022

Licensee PAGEPress, Italy

Journal of Biological Research 2022; 95:10259

doi:10.4081/jbr.2022.10259

This article is distributed under the terms of the Creative Commons Attribution Noncommercial License (by-nc 4.0) which permits any noncommercial use, distribution, and reproduction in any medium, provided the original author(s) and source are credited. both quantity and quality of SFMC composition compared to that of healthy subjects. Our results also demonstrated the elevated level of high molecular weight Vitamin K-Dependent Protein (VKDP) complexes in plasma of CAD patients, which could be used as a potential biomarker of hypercoagulation state. More studies are clearly needed to investigate whether SFMCs or abnormal forms of VKDPs affect cardiovascular events under CAD development and progression.

\section{Introduction}

Coronary Artery Disease (CAD), also called coronary heart disease, is the leading cause of death in both men and women, accounting for about one third of all deaths. CAD is caused by the narrowing of the coronary arteries that supply the heart with oxygen. ${ }^{1}$ The reduced blood flow may cause shortness of breath, chest pain (angina), and lead to other health problems like heart failure or heart rhythm problems. A complete blockage of a coronary artery can result in a heart attack. ${ }^{2}$ A heart attack, also called a Myocardial Infarction (MI), is a major cause of mortality, with most deaths occurring prior to arrival to the hospital. At least 5\%$10 \%$ of survivors die within the first 12 months after the MI, and close to $50 \%$ need hospitalization within the same year. ${ }^{3}$

Clinical understanding of the pathophysiology underlying $\mathrm{CAD}$ has greatly increased in recent years, but there remains a lack of knowledge regarding the exact factors that determine the severity of an acute coronary event and its long-term outcomes. It is recognized that acute coronary syndromes are caused mainly by coronary thrombi ${ }^{4,5}$ and only in rare cases by spasm of coronary artery. ${ }^{6}$ Because clotting is relevant to the development of CAD, it is reasonable to expect that abnormalities of the coagulation system affect the risk of future cardiovascular events. Indeed, an increase in the concentration and activity of a number of individual coagulation factors have been noted in patients with angina pectoris and in post-infarction patients. ${ }^{7-9}$ Given the fact that blood coagulation factors could be involved in the initiation of coronary thrombus formation, and progression of vascular complications during CAD, the monitoring of the key parameters of hemostasis system might have a high diagnostic value to improved patient management resulting in better clinical outcomes.

The notion that fibrinogen is a major cardiovascular risk factor has been widely accepted. The evidence is based on numerous prospective epidemiological studies and clinical observations. ${ }^{10,11}$ Apart from its pivotal role in the coagulation cascade as the substrate for thrombin, there is evidence of multiple mechanisms suggesting that fibrinogen indeed may be involved in both the early and the later stages of the thrombotic process. ${ }^{12,13}$ However, the pathophysiological mechanisms by which fibrinogen is involved in thrombogenesis still remains to be determined. A recent study by Yoshihisa et al. ${ }^{14}$ has revealed that the high level of Soluble Fibrin 
Monomer Complexes (SFMCs), produced by thrombin-mediated cleavage of fibrinogen in a hypercoagulable state, was associated with the risk of adverse cardiovascular events and could be considered as a very sensitive biomarker for prediction of thrombotic events in heart failure patients. On the other hand, in other study ${ }^{15}$ it was shown that SFMC levels did not predict subsequent thromboembolic events. Thus, the association between SFMC and cardiovascular events in CAD patients remains uncertain. We believe that it makes sense to pay attention not only to the total SFMC content but to the composition of SFMC faction in patients with CAD.

Given the above, the aim of this study was to investigate some coagulation parameters, including fibrinogen, prothrombin, the vitamin K-dependent coagulation factors, and to examine the composition of SFMC fractions collected from blood plasma of healthy volunteers, and patients with stable angina, unstable angina, and MI.

\section{Materials and Methods}

\section{Selection of study groups}

The study included 88 patients who were hospitalized to the cardiology department of Kyiv City Hospital \#12 with a preliminary diagnosis of CAD. We followed the guidelines of European Society of Cardiology $2020^{16}$ to establish the diagnosis and manage the patients. Thus, MI was confirmed by ECG, a diagnostic level of cardiospecific biomarkers (troponin T, CK-MB) and coronary angiography with the presence of one infarct-related coronary artery. Chronic stable coronary angina patients confirmed by invasive coronary angiography. Furthermore, stable angina and unstable angina patients were characterized by following clinical guidelines of ACC/AHA. ${ }^{17,18}$ After the full set investigation was completed, we defined four groups: group I - Stable Angina patients (SA, $n=25$ ), group II - patients with unstable angina pectoris of new onset (NOUA, n=17), group III - patients with Progressive Unstable Angina (PUA, $\mathrm{n}=28$ ), and group IV - patients with Myocardial Infarction (MI, $\mathrm{n}=18$ ). It should be noted that patients with less than 45 years and more than 75 years old, chronic inflammatory diseases, autoimmune diseases, acute infectious diseases, chronic liver and kidney diseases were not enrolled in this research. The control group included 20 healthy volunteers. Healthy controls were well matched with age, gender, and other basic information with patients' group and also free from cardiovascular and any chronic disease.

This study has been followed all the principles outlined by the revised Helsinki Declaration in 2013 for human subjects. The study design was reviewed and approved by the Ethical Committees of both the Kyiv City Hospital \#12 and Taras Sevchenko University of Kyiv. All patients or their relatives have given their written informed consent, after having been informed of all aspects of the particular trial.

\section{Collection of blood samples and plasma preparation}

The blood from patients was drawn within $2 \mathrm{~h}$ after hospitalization, before treatment onset. Plasma for analysis was obtained from blood samples collected from the cubital vein in standard plastic vacuum tubes with $3.8 \%$ sodium citrate. The volume ratio of blood to citrate was equal to 9:1. Plasma was prepared by centrifugation of blood for $25 \mathrm{~min}$ at $2500 \mathrm{~g}$.

\section{Biochemical assays}

A set of routine laboratory tests, including lipid profile, and total protein, was performed by the laboratory of the hospital.
Cardiac troponin I (cTnI) was analyzed by chemiluminescent immunoassay (reference ranges: $<7 \mathrm{pg} / \mathrm{mL}$ for males, and $<5$ $\mathrm{pg} / \mathrm{mL}$ - for females). Body mass index (BMI) was calculated as weight in kilograms divided by height in meters squared.

Plasma fibrinogen concentration was estimated by the Clauss method using commercial kit of reagents (Renam, Rassia). Concentration of Soluble Fibrin Monomer Complexes (SFMCs) in blood plasma was measured by $o$-phenanthroline method using commercial kit of reagents (Renam, Rassia).

Plasma prothrombin level was measured by means of ELISA technique, using corresponding primary antibody (Santa Crus Biotechnology, CA, USA), secondary antibody conjugated with horseradish peroxidase (Sigma, USA), and $o$-phenylenediamine (Sigma, USA) as a chromogenic substrate. All procedures were done according to the standard protocol instructions as described previously. ${ }^{19}$

\section{Isolation and chromatographic separation of Soluble Fibrin Monomer Complexes (SFMCs)}

The isolation of SFMCs from blood plasma was performed according to the procedure described previously. ${ }^{20,21}$ The SFMCs were collected from $1 \mathrm{~mL}$ of blood plasma of each individual and kept at $4^{\circ}$ till chromatographic analysis. Before chromatographic analysis, equal volume of SFMCs solutions obtained from five individuals of the same experimental group were randomly gathered to form pool of SFMCs, which was used in further analysis.

Size-exclusion chromatography was performed on Superdex 200 pg column (HiLoad 16/60, Healthcare Life Sciences), using a BioLogic LP chromatographer (BioRad, USA). The mobile phase was $0.05 \mathrm{M}$ Tris- $\mathrm{HCl}(\mathrm{pH} 7.4)$ containing $0.13 \mathrm{M} \mathrm{NaCl}$, and the flow rate was $60 \mathrm{~mL} / \mathrm{hr}$. The pool of SFMCs gathered from five individuals was loaded onto the column in the volume of $1 \mathrm{~mL}$, and the appearance of corresponding peaks (fractions) were monitored by means of UV detector. The peak areas were calculated using OriginLab (v 9.1). The chromatographic procedure was repeated at least three times for each experimental group. Every time we used freshly prepared SFMCs pool solution obtained by mixing of five different SFMC fractions isolated from individuals of the same experimental group. A standard ladder with known molecular weight was applied to the column for calibration.

\section{Separation of vitamin K-dependent coagulation proteins}

The fraction of vitamin K-dependent coagulation proteins was obtained from plasma according to the procedure described previously. ${ }^{21}$ Briefly, $30 \mathrm{mg}$ of $\mathrm{BaSO}_{4}$ was added into $1 \mathrm{~mL}$ of blood plasms, and the mixture was placed on ice for 1 hour, then centrifuged at $2000 \mathrm{~g}$ for $15 \mathrm{~min}$. Elution of vitamin K-dependent proteins from the precipitate was done by adding of $50 \mathrm{mM}$ Tris- $\mathrm{HCl}$ buffer, $\mathrm{pH} 7.4$, containing $200 \mathrm{mM} \mathrm{NaCl}$ and $20 \mathrm{mM}$ EDTA.

Chromatographic analysis of vitamin K-dependent protein fractions was done according to the same procedure described above.

\section{Statistical analysis}

Statistical analysis was performed with Statistica 8.0 software. All results were expressed as mean \pm SD. The KolmogorovSmirnov test was used to verify the normal distribution of results. Nonparametric data were analyzed using the Mann-Whitney test when comparing two groups and the Kruskal-Wallis test followed by Dunn's post-test for comparing three or more groups. The statistical significance was determined as $\mathrm{p}<0.05$. 


\section{Results}

Basic clinical characteristics of patients with CAD are shown in Table 1. No significant differences in lipid profile and total protein content were observed between the patients with stable and both groups of unstable angina pectoris. In contrast, patients with MI have significantly increased level of total glycerides, and decreased level of high-density lipoproteins. The concentration of total protein was also significantly elevated in patients with MI, compared to control subjects. The plasms level of cardiac troponin $\mathrm{I}$, which is the recommended biomarker to identify myocardial injury, rose in patients with acute MI (Table 1).

One of the most important plasma proteins that are critical for hemostasis and clot formation is fibrinogen. It is broken down to fibrin by the thrombin (coagulation factor II) providing the structural network required to form clot. The normal value for fibrinogen in blood plasma is $2-4 \mathrm{~g} / \mathrm{L}$, with levels appreciably below or above this range associated with such pathological conditions as bleeding or excessive blood clotting. Since elevated fibrinogen level is considered a promising biomarker of cardiovascular risk, we have examined the fibrinogen concentration in plasma of patients with CAD. The results showed that there were no significant differences between fibrinogen concentrations in plasma of patients with angina pectoris, and control subjects. However, patients suffering from myocardial infarction have been shown to have increased fibrinogen values (Table 2).

We also estimated the plasma prothrombin concentration that is a marker of the in vivo rate of generation of thrombin enzyme involved in the final step of the coagulation cascade. Our findings revealed that prothrombin levels were similar in plasma of all groups of patients with $\mathrm{CAD}$, and were significantly decreased compared to control subjects (Table 2). The decreased level of prothrombin might be explained by its conversion to thrombin. Once activated, thrombin triggers fibrinogen polymerization into fibrine, leading to plug formation.

The other important biomarker of cardiovascular events is SFMC, which is a precursor of fibrin polymer formation at the early stage of blood coagulation. Thus, the appearance of such molecular complexes is expected to serve as a promising biomarker for early detection and diagnosis of intravascular hypercoagulation condition.

To determine the content of soluble fibrin monomer complexes, $o$-phenanthroline test was used. This method unlike the widely used ethanol and protamine sulfate test is more informative, standardized and has great diagnostic value. ${ }^{22,23}$ The results are shown in Table 2. As can be seen from the data obtained there were significant differences between SFMC concentrations in plasma of patients with CAD and control subjects. The concentration of SFMC in plasma of healthy volunteers was $2.0 \pm 0.5 \mathrm{~g} / \mathrm{L}$. In patients with unstable angina of both types, new onset and progressive, as well as in patients with MI, the plasma concentrations of SFMC were almost the same, and ranged from $18.1 \times 10^{-2} \mathrm{~g} / \mathrm{L}$ to $28.1 \times 10^{-2} \mathrm{~g} / \mathrm{L}$, that was almost 12 -fold above control values. The concentration of SFMC in plasms of patients with stable angina was lower compared to other groups of patients with $\mathrm{CAD}$ but significantly higher than in control subjects.

Since our findings indicated that SFMC levels have been significantly elevated under cardiovascular events, we aimed to analyze and compare the composition of SFMC factions isolated from plasma of patients with CAD and healthy volunteers.

Thus, at the next step of our study, equal amounts of samples of SFMC separated from $1 \mathrm{~mL}$ of plasma of 5 different individu-

Table 1. The main clinical characteristics of patients with $\mathrm{CAD}$, and control subjects.

\begin{tabular}{|c|c|c|c|c|c|}
\hline & Control $(n=20)$ & SA $(n=25)$ & NOUA $(n=17)$ & PUA $(n=28)$ & MI $(n=18)$ \\
\hline Age, years & $53 \pm 6$ & $63 \pm 8$ & $62 \pm 6$ & $67 \pm 7$ & $61 \pm 7$ \\
\hline Sex, male, n (\%) & $10(50)$ & $15(60)$ & $11(65)$ & $14(50)$ & $12(70)$ \\
\hline $\mathrm{BMI}, \mathrm{kg} / \mathrm{m}^{2}$ & $27 \pm 4$ & $29 \pm 6$ & $28 \pm 3$ & $29 \pm 3$ & $28 \pm 6$ \\
\hline $\mathrm{TC}, \mathrm{mmol} \cdot \mathrm{L}^{-1}$ & $5.14 \pm 0.99$ & $5.96 \pm 1.64$ & $4.90 \pm 0.75$ & $6.39 \pm 2.18$ & $5.28 \pm 0.94$ \\
\hline $\mathrm{TG}, \mathrm{mmol} \cdot \mathrm{L}^{-1}$ & $1.28 \pm 0.43$ & $2.50 \pm 0.70$ & $1.89 \pm 0.85$ & $2.21 \pm 1.12$ & $3.29 \pm 1.17^{*}$ \\
\hline $\mathrm{LDL}, \mathrm{mmol} \cdot \mathrm{L}^{-1}$ & $2.38 \pm 0.47$ & $3.39 \pm 1.38$ & $2.83 \pm 0.62$ & $3.75 \pm 1.19$ & $3.29 \pm 1.17$ \\
\hline $\mathrm{HDL}, \mathrm{mmol} \cdot \mathrm{L}^{-1}$ & $1.64 \pm 0.45$ & $1.64 \pm 0.87$ & $1.22 \pm 0.36$ & $1.31 \pm 0.51$ & $0.85 \pm 0.24^{*}$ \\
\hline TP, $g \cdot L^{-1}$ & $13.8 \pm 4.0$ & $10.4 \pm 5.0$ & $11.7 \pm 3.4$ & $11.5 \pm 4.6$ & $35.5 \pm 11.8^{*}$ \\
\hline hs-cTnI, pg.mL ${ }^{-1}$ & $\leq 5$ & $\leq 5$ & $8.1 \pm 4.3$ & $17.6 \pm 9.2^{*}$ & $24.3 \pm 10.2^{*}$ \\
\hline
\end{tabular}

Values are expressed as mean $\pm \mathrm{SD} ;{ }^{*} \mathrm{p}<0.05$ significantly different from control subjects; $\mathrm{TC}=$ total cholesterol, $\mathrm{TG}=$ total glycerides, $\mathrm{LDL}=$ low density lipoproteins, $\mathrm{HDL}=$ high density lipoproteins, TP = total protein, hs-cTnI = high sensitive cardiac tropolin I.

Table 2. Some plasma coagulation parameters in patients with CAD, and control subjects.

\begin{tabular}{|c|c|c|c|}
\hline Groups & Fibrinogen, $g \cdot L^{-1}$ & Prothrombin, units $\cdot \mathrm{mL}^{-1}$ & SFMC, $\times 10^{-2} \mathrm{~g} \cdot \mathrm{L}^{-1}$ \\
\hline Control $(\mathrm{n}=20)$ & $2.4 \pm 0.6$ & $0.26 \pm 0.04$ & $2.0 \pm 0.5$ \\
\hline SA $(n=25)$ & $2.8 \pm 0.7$ & $0.15 \pm 0.04^{*}$ & $12.7 \pm 2.8^{*}$ \\
\hline NOUA $(\mathrm{n}=17)$ & $3.3 \pm 0.6$ & $0.14 \pm 0.03^{*}$ & $25.3 \pm 2.8^{*}$ \\
\hline PUA $(n=28)$ & $3.2 \pm 0.5$ & $0.15 \pm 0.03^{*}$ & $24.1 \pm 4.1^{*}$ \\
\hline $\mathrm{MI}(\mathrm{n}=17)$ & $5.4 \pm 0.5^{*}$ & $0.14 \pm 0.02 *$ & $22.6 \pm 4.5^{*}$ \\
\hline
\end{tabular}

Values are expressed as mean $\pm \mathrm{SD} ;{ }^{*} \mathrm{p}<0.05$ significantly different from control subjects. 
als within the same group were randomly gathered, and the pool of SFMCs was subjected to further characterization. Size exclusion chromatography was applied successfully to separate molecular complexes of different size under native conditions. Zones of different size protein fractions releasing were monitored by means of UV sensor $(280 \mathrm{~nm})$. A typical chromatogram of SFMCs separation on Superdex $200 \mathrm{pg}$ column is shown at Figure 1. Each peak represents a protein fraction with particular molecular weight that is present in the SFMC sample. To identify the molecular weight of each fraction, a set of standards (protein ladders with known molecular weights) was applied to the column for calibration. The estimated molecular weight values and other quantitative properties that were detected by column calibration are listed in Table 3.

Our findings revealed the presence of a number of protein molecules with different molecular weight (up to $865 \mathrm{kDa}$ ) in the tested SFMC pools. The difference between the results of fractiona-

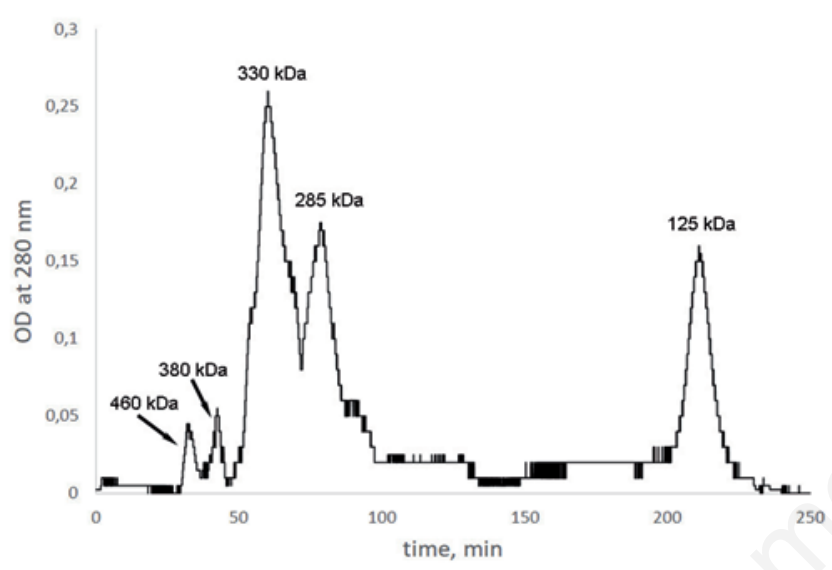

Figure 1. A typical chromatogram of separation of total SFMCs fraction obtained from the blood plasma on Superdex $200 \mathrm{pg}$ column in $0.05 \mathrm{M}$ Tris-HCl, pH 7.4, containing $0.13 \mathrm{M} \mathrm{NaCl}$; flow rate: $1 \mathrm{~mL} / \mathrm{min}$. Molecular weight of the protein fractions was estimated by the same gel filtration column, and under the same conditions, with thyroglobulin, ferritin, catalase, and aldolase as the molecular weight standards. tion of SFMC obtained from plasma of CAD patients and healthy donors was obvious. On the other hand, the trend of the SFMC content was similar to all tested CAD groups (Table 3 ).

As can be seen from the Table 3, the SFMC pool of healthy subjects consists of five fractions. The most abundant protein fraction in SFMC pool of healthy individuals was fraction with molecular weight of $330-350 \mathrm{kDa}$, and represented about $40 \%$ of total SFMCs. The levels of two other fractions, $125-130 \mathrm{kDa}$, and 285$320 \mathrm{kDa}$ were almost equal, each of them represented about $24 \%$ of total SFMC pool. Finally, $10 \%$ of SFMCs was represented by 380-390 kDa, and 450-460 kDa protein complexes.

The development of CAD pathologies was accompanied by the changes in both quantity and quality composition of SFMC pool compared to the results obtained for healthy subjects (Table 3). Thus, in additional to those fractions precent in SFMC pool of healthy subjects, fractions with higher molecular weight were observed. Protein complexes with molecular weight of 670-690 kDa were identified in plasma of patients of all CAD groups tested: trace amount (4-7\%) was observed in SA, NOSA, and MI groups, while in PUA patients this fraction represented $20 \%$ of total SFMC pool.

Based on the results of our study, we have concluded that there is dramatic dysregulation of coagulation process associated with prothrombin activation, and fibrinogen cleavage in patients with CAD. Considering these findings, we hypothesized that abnormal forms of coagulation factors and/or their complexes, which appear under hypercoagulation state, might have a role in cardiovascular pathophysiology. Thus, the next step of our research was to analyze the content of plasma Vitamin K-Dependent Proteins (VKDPs) that include coagulant factors II, VII, IX, and X and the anticoagulants protein $\mathrm{C}$ and protein $\mathrm{S}$. Because of their similar structures, they tend to co-purify by most of the methods used to isolate them from plasma. In our study, the VKDPs were adsorbed from plasma using barium sulfate. Later, pool of VKDPs was fractionated by size-exclusion chromatography.

As can be seen from the Table 4, the most abundant VKDP fraction in plasma of healthy subjects was fraction with molecular weight of $55-70 \mathrm{kDa}$, which represented about $80 \%$ of total pool. This fraction may include the zymogen procoagulant factors VII, IX, $\mathrm{X}$, and prothrombin, and the anticoagulants protein $\mathrm{C}$ and protein $\mathrm{S}$, since they all have similar molecular weight ranging from 55 to $72 \mathrm{kDa}$. Our results revealed the presence of a small amount (about 13\% of total VKDPs) of protein molecules with molecular

Table 3. Some characteristics of individual SFMC fractions derived from the plasma of patients with CAD, and control subjects.

\begin{tabular}{|c|c|c|c|c|c|}
\hline $\begin{array}{l}\text { Molecular weight } \\
\text { rang, kDa }\end{array}$ & Control $(n=20)$ & $\begin{array}{r}\text { SA }(\mathrm{n}=25) \\
\text { Area }\end{array}$ & $\begin{array}{l}\text { NOUA }(n=17) \\
\text { individual peal }\end{array}$ & $\begin{array}{l}\text { PUA }(n=28) \\
n \text { total }\end{array}$ & MI $(n=17)$ \\
\hline $855-865$ & - & - & - & $6 \pm 2$ & - \\
\hline $670-690$ & - & $4 \pm 1$ & $6 \pm 2$ & $20 \pm 3^{*}$ & $7 \pm 2$ \\
\hline $595-615$ & - & - & - & - & $11 \pm 3^{*}$ \\
\hline $520-530$ & - & $10 \pm 1^{*}$ & - & - & - \\
\hline $450-460$ & $5 \pm 1$ & $28 \pm 3^{*}$ & $30 \pm 3^{*}$ & $26 \pm 3^{*}$ & $22 \pm 2^{*}$ \\
\hline $380-390$ & $5 \pm 1$ & $12 \pm 1^{*}$ & $15 \pm 2^{*}$ & $11 \pm 2^{*}$ & $3 \pm 2^{*}$ \\
\hline $330-350$ & $41 \pm 2$ & $18 \pm 2^{*}$ & $13 \pm 2^{*}$ & $5 \pm 2^{*}$ & $18 \pm 2^{*}$ \\
\hline 285-320 & $26 \pm 3$ & - & $8 \pm 2^{*}$ & $17 \pm 1$ & - \\
\hline $255-265$ & - & - & - & - & $26 \pm 3^{*}$ \\
\hline $210-215$ & - & - & $7 \pm 2$ & - & - \\
\hline $125-130$ & $23 \pm 2$ & $28 \pm 3$ & $21 \pm 3$ & $15 \pm 2 *$ & $13 \pm 1^{*}$ \\
\hline
\end{tabular}

Values are expressed as mean $\pm \mathrm{SD} ;{ }^{*} \mathrm{p}<0.05$ significantly different from control subjects. 
weight of $145-150 \mathrm{kDa}$, that is twice-higher than molecular weight of typical coagulation factors (Table 4). We believe, this fraction consists of protein complexes that could be formed in the dynamic process of coagulation to maintain the hemostatic balance. For example, such molecular complexes might be formed in the resalts of cooperation of active coagulation factor and its inhibitor.

The development of CAD pathologies was accompanied by the changes in quantity of these two major fractions compared to the results obtained for control subjects. Thus, the contents of vitamin $\mathrm{K}$-dependent coagulation factors with molecular weight about 55$70 \mathrm{kDa}$ have been progressively decreasing under the progression of CAD symptoms from stable angina to unstable angina, and MI. Furthermore, the elevated level of high molecular weight complexes of VKDPs appears to corelate with more severe form of CAD.

\section{Discussion}

A total of 88 patients were included in the study; $60 \%$ of the participants were men. Overall, the mean $( \pm \mathrm{SD})$ age of participants was $64 \pm 6$ years. Four groups were created based on the clinical data: group I - stable angina patients $(n=25)$, group II - patients with unstable angina of new onset $(n=17)$, group III - patients with progressive unstable angina $(n=28)$, and group IV - patients with myocardial infarction $(\mathrm{n}=18)$. We also analyzed individuals $(\mathrm{n}=20)$ without a history of cardiovascular disease. Our aim was to estimate some key parameters of coagulation system in patients with cardiovascular diseases. Our principal focus was analysis of composition of SFMC factions derived from plasma of patients with $\mathrm{CAD}$ and healthy voluntaries in order to determine whether a positive SFMC test could be used as a biomarker of cardiovascular events. The second focus of our research was to analyze the content of vitamin K-dependent coagulation factors and their complexes under CAD development.

Major differences between healthy volunteers and CAD patients were as follows: i) fibrinogen level was 2-fold higher in patients with MI compared to control subjects; ii) concentration of SFMC in SA patients was 6-fold higher, and about 12-fold higher in the other tested CAD groups than in control subjects; iii) concentration of prothrombin was lower by $40 \%$ in plasma of CAD patients of all studied groups compared to control subjects; iv) the levels of high molecular weight SFMCs as well as complexes of VKDPs were significantly increased in plasma of patients with CAD compared to control subjects.

Our study confirms the findings of other researchers ${ }^{24,25}$ that fibrinogen levels are elevated after acute myocardial infarction. Since fibrinogen level reflects the balance between the various procoagulants and lytic processes in the body, we can suggest that the rise seen in MI group of patients could be due to decreased blood-lytic potential, in addition to being a response to non-specific tissue damage.

The elevated SFMC level appears to be related to activation of coagulation cascade. When thrombin cleaves one or both of A or B peptides of fibrinogen, the fibrin monomers are formed, which, in the early stages of thrombus formation, attain stability in plasma by forming SFMCs with fibrinogen degradation products. As the process of thrombosis continues, more fibrin monomers are created and more complexes are formed. When concentration is reached threshold level fibrin polymers are created, and interact with factor XIII to form stable clots. Since SFMCs can be detected before actual "clot" formation occurs, these molecular complexes could be used as a biomarker of increased level of intravascular coagulation in its early stages. Returning to the hypothesis posed at the beginning of this study, it is now possible to state that test for SFMC plasma level has clinical significance in patients with CAD, and elevated level of these molecular complexes could be implicated as a risk factor for cardiovascular events. Furthermore, an increased SFMC level is typical for the development of ischemic stroke, disseminated intravascular coagulation, autoimmune diseases and thrombophilia. ${ }^{14,21,26}$

Our further analysis of composition of SFMCs derived from plasma of CAD patients revealed the appearance of protein complexes with molecular weight that differ from that of healthy subjects. We believe that alteration in SFMC composition might be due the abnormalities of coagulation process under pathological conditions. The formation of high molecular weight complexes might be associated with enhanced plasma coagulation in CAD patients.

Similar conclusion has also been reached in case of analysis of vitamin K-dependent coagulation factors. The presence in plasma VKDPs with molecular weights more than $75 \mathrm{kDa}$ could be result of the formation of complexes of prothrombin and its derivative with other proteins at the term of CAD progression. The appearance in the bloodstream of high molecular weight complexes seems to be one of the diagnostic criteria for cardiovascular events development.

\section{Conclusions}

The obtained results have shown that coronary artery diseases, such as stable angina, unstable angina, and myocardial infarction, are characterized by similar changes of the investigated hemostasis parameters. An increased SFMC plasma level, and the presence of high molecular weight complexes of VKDPs appear to be associated with increased risk of cardiovascular events. Studies are cur-

Table 4. Some characteristics of individual vitamin K-dependent protein fractions derived from the plasma of patients with CAD, and control subjects.

\begin{tabular}{|c|c|c|c|c|c|}
\hline $\begin{array}{l}\text { Molecular weight } \\
\text { rang, kDa }\end{array}$ & Contro $1(n=20)$ & SA $(n=25)$ & $\begin{array}{l}\text { NOUA }(n=17) \\
\text { er the peak, \% }\end{array}$ & PUA $(n=28)$ & MI $(n=17)$ \\
\hline $181-189$ & $3 \pm 1$ & $2 \pm 1$ & $5 \pm 2$ & $4 \pm 1$ & $4 \pm 1$ \\
\hline $145-150$ & $13 \pm 3$ & $36 \pm 3^{*}$ & $40 \pm 2 *$ & $49 \pm 2 *$ & $42 \pm 3^{*}$ \\
\hline $125-130$ & $5 \pm 2$ & $4 \pm 2$ & $7 \pm 1$ & $6 \pm 2$ & $7 \pm 2$ \\
\hline $77-80$ & - & - & $5 \pm 2$ & $5 \pm 1$ & $5 \pm 2$ \\
\hline $55-70$ & $79 \pm 3$ & $58 \pm 3^{*}$ & $43 \pm 1^{*}$ & $37 \pm 2^{*}$ & $42 \pm 1^{*}$ \\
\hline
\end{tabular}

Values are expressed as mean $\pm \mathrm{SD} ;{ }^{*} \mathrm{p}<0.05$ significantly different from control subjects. 
rently underway to determine the long-term prognostic significance of persistently elevated level of high molecular weight SFMCs and VKDP complexes in patients with coronary heart disease. Theoretically, if associated with defective fibrinolytic activity, it would carry a grave prognostic value.

\section{References}

1. Malakar AK, Choudhury D, Halder B, et al. A review on coronary artery disease, its risk factors, and therapeutics. J Cell Physiol 2019;234:16812-23.

2. Lu L, Liu M, Sun R, et al. Myocardial infarction: symptoms and treatments. Cell Biochem Biophys 2015;72:865-7.

3. Law MR, Watt HC, Wald NJ. The underlying risk of death after myocardial infarction in the absence of treatment. Arch Intern Med 2002;162:2405-10

4. Falk E, Nakano M, Bentzon JF, et al. Update on acute coronary syndromes: the pathologists' view. Eur Heart J 2013;34:719-28.

5. Libby P, Theroux P. Pathophysiology of coronary artery disease. Circulation 2005;111:3481-8.

6. Hung MY, Kounis NG, Lu MY, Hu P. Myocardial ischemic syndromes, heart failure syndromes, electrocardiographic abnormalities, arrhythmic syndromes and angiographic diagnosis of coronary artery spasm: literature review. Int J Med Sci 2020;17:1071-82.

7. Rennie JA, Ogston D. Changes in coagulation factors following acute myocardial infarction in man. Haemostasis 1976;5:258-64.

8. Minnema MC, Peters RJ, de Winter R, et al. Activation of clotting factors XI and IX in patients with acute myocardial infarction. Arterioscler Thromb Vasc Biol 2000;20:2489-93.

9. Vaziri ND, Kennedy SC, Kennedy D, Gonzales E. Coagulation, fibrinolytic, and inhibitory proteins in acute myocardial infarction and angina pectoris. Am J Med 1992;93:651-7.

10. Koenig W. Fibrin(ogen) in cardiovascular disease: an update. Thromb Haemost 2003;89:601-9.

11. Lind P, Hedblad B, Stavenow L, et al. Influence of plasma fibrinogen levels on the incidence of myocardial infarction and death is modified by other inflammation-sensitive proteins: a long-term cohort study. Arterioscler Thromb Vasc Biol 2001;21:452-8.

12. Schneider DJ, Taatjes DJ, Howard DB, Sobel BE. Increased reactivity of platelets induced by fibrinogen independent of ist bindings to the IIb-IIIa surface glycoprotein. A potential contributor to cardiovascular risk. J Am Coll Cardiol 1999;33:261-6.

13. Fatah K, Hamsten A, Blomback B, Blomback M. Fibrin gel network characteristics and coronary heart disease: Relations to plasma fibrinogen concentration, acute phase protein, serum lipoproteins and coronary atherosclerosis. Thromb Haemost 1992; 68:130-5.

14. Yoshihisa A, Sato Y, Kimishima Y, et al. Soluble fibrin monomer complex is associated with cardio- and cerebrovascular events in patients with heart failure. Int J Cardiol Heart Vasc 2020;32:100697.
15. Sadanaga T, Mitamura H. Soluble fibrin monomer complex levels during oral anticoagulant therapy do not predict subsequent thromboembolic events in patients with permanent atrial fibrillation. Int J Cardiol 2013;168:578-80.

16. Collet JP, Thiele H, Barbato E, et al. 2020 ESC Guidelines for the management of acute coronary syndromes in patients presenting without persistent ST-segment elevation. Eur Heart J 2021; 42:1289-1367.

17. Fihn SD, Blankenship JC, Alexander KP, et al. 2014 ACC/AHA/AATS/PCNA/SCAI/STS focused update of the guideline for the diagnosis and management of patients with stable ischemic heart disease: a report of the American College of Cardiology/American Heart Association Task Force on Practice Guidelines, and the American Association for Thoracic Surgery, Preventive Cardiovascular Nurses Association, Society for Cardiovascular Angiography and Interventions, and Society of Thoracic Surgeons. J Am Coll Cardiol 2014;64:1929-49.

18. Amsterdam EA, Wenger NK, Brindis RG, et al. 2014 AHA/ACC Guideline for the Management of Patients with Non-ST-Elevation Acute Coronary Syndromes: a report of the American College of Cardiology/American Heart Association Task Force on Practice Guidelines. J Am Coll Cardiol 2014;64:e139-e228.

19. Kemeny M. A Practical Guide to ELISA. Oxford: Pergamon Press; 1991.

20. Katrii T, Shabanova N, Ostapchuk S, Savchuk O. The characterization of the soluble fibrin monomer complex in patients with acute and one year post acute ischemic stroke. Bulletin of Taras Shevchenko National University of Kyiv: Series "Problems of Physiological Functions Regulation" 2017;1:58-61.

21. Katrii TB, Vovk TB, Halenova TI, et al. Difference in coagulation markers in acute and one year post acute ischemic stroke. Period Biol 2017;119:189-98.

22. Hosaka A, Miyata T, Aramoto H, et al. Clinical implication of plasma level of soluble fibrin monomer-fibrinogen complex in patients with abdominal aortic aneurysm. J Vasc Surg 2005; 42:200-5.

23. Momot AP, Elykkomov VA, Barkagan ZS. Metodika i klinicheskoe znachenie parakoaguliatsionnogo fenantrolinovogo testa [Methods and clinical significance of paracoagulation phenanthroline test]. Klin Lab Diagn 1996;4:17-20.

24. Gil M, Zarebiński M, Adamus J. Plasma fibrinogen and troponin $\mathrm{I}$ in acute coronary syndrome and stable angina. Int $\mathrm{J}$ Cardiol 2002;83:43-6.

25. Ang L, Behnamfar O, Palakodeti S, et al. Elevated baseline serum fibrinogen: effect on 2-year major adverse cardiovascular events following percutaneous coronary intervention. J Am Heart Assoc 2017;6:e006580.

26. Ieko M, Naito S, Yoshida M, et al. Plasma soluble fibrin monomer complex as a marker of coronary thrombotic events in patients with acute myocardial infarction. Tohoku J Exp Med 2009; 219:25-31. 